\title{
Evidence generation for the clinical impact of myCOPD in patients with mild, moderate and newly diagnosed COPD: a randomised controlled trial
}

\author{
Michael G. Crooks ${ }^{1}$, Jack Elkes (10) ${ }^{2}$, William Storrar ${ }^{3}$, Kay Roy ${ }^{4}$, Mal North ${ }^{5}$, \\ Alison Blythin (10 ${ }^{5}$, Alastair Watson (10) ${ }^{6}$, Victoria Cornelius (10) ${ }^{2}$ and \\ Tom M.A. Wilkinson ${ }^{5,6}$
}

ABSTRACT Self-management interventions in COPD aim to improve patients' knowledge, skills and confidence to make correct decisions, thus improving health status and outcomes. myCOPD is a webbased self-management app known to improve inhaler use and exercise capacity in individuals with more severe COPD.

We explored the impact of myCOPD in patients with mild-moderate or recently diagnosed COPD through a 12-week, open-label, parallel-group, randomised controlled trial of myCOPD compared with usual care. The co-primary outcomes were between-group differences in mean COPD assessment test (CAT) score at 90 days and critical inhaler errors. Key secondary outcomes were app usage and patient activation measurement (PAM) score.

Sixty patients were randomised (29 myCOPD, 31 usual care). Groups were balanced for forced expiratory volume in $1 \mathrm{~s}\left(\mathrm{FEV}_{1} \%\right.$ pred) but there was baseline imbalance between groups for exacerbation frequency and CAT score. There was no significant adjusted mean difference in CAT score at study completion, -1.27 (95\% CI -4.47-1.92, $\mathrm{p}=0.44$ ) lower in myCOPD. However, an increase in app use was associated with greater CAT score improvement. The odds of $\geqslant 1$ critical inhaler error was lower in the myCOPD arm (adjusted OR 0.30 (95\% CI 0.09-1.06, $\mathrm{p}=0.061)$ ). The adjusted odds ratio for being in a higher PAM level at 90 days was 1.65 (95\% CI 0.46-5.85) in favour of myCOPD.

The small sample size and phenotypic difference between groups limited our ability to demonstrate statistically significant evidence of benefit beyond inhaler technique. However, our findings provide important insights into associations between increased app use and clinically meaningful benefit warranting further study in real world settings.

@ERSpublications

A preliminary trial of a self-management intervention using a scalable, @NHS-approved app demonstrated signals of potential clinical benefit in a population of patients with mild-moderate and newly diagnosed \#COPD over a 90-day period https://bit.ly/2PNDR8A

Cite this article as: Crooks MG, Elkes J, Storrar W, et al. Evidence generation for the clinical impact of myCOPD in patients with mild, moderate and newly diagnosed COPD: a randomised controlled trial. ERJ Open Res 2020; 6: 00460-2020 [https://doi.org/10.1183/23120541.00460-2020].

This article has supplementary material available from https:/openres.ersjournals.com/

This study is registered at www.clinicaltrials.gov with identifier number NCT03620630. All relevant data have been included within the manuscript. A full statistical analysis plan was written prior to data extraction and, alongside additional data, is available upon request.

The trial received UK research ethics committee and Health Research Authority approval (REC 18/SS/0112).

Received: 2 July 2020 | Accepted after revision: 6 Aug 2020

Copyright $\odot$ ERS 2020. This article is open access and distributed under the terms of the Creative Commons Attribution Non-Commercial Licence 4.0. 


\section{Introduction}

Chronic obstructive pulmonary disease (COPD) is a common and preventable disease and is predicted to become the 3rd leading cause of death by 2030 [1]. There are an estimated 1.2 million sufferers in the UK [2] and around 80000 people are diagnosed with COPD each year in England [3, 4].

Despite prescribed therapy, COPD continues to drive excessive morbidity and is a leading cause of hospital admission $[2,5,6]$. COPD self-management interventions aim to combat this by helping patients acquire knowledge, skills and confidence to make the correct decisions about treatment, lifestyle and health behaviours. Self-management interventions that improve health-related quality of life and reduced hospitalisations have traditionally been delivered face-to-face [7].

Applications (apps) and websites have been developed to support COPD self-management. Digital solutions are attractive because they can be implemented long-term, are low-cost and can be used by large numbers of people at their convenience [8]. However, adoption can be hindered by low digital literacy, limited perceived benefit and/or poor alignment with illness or social context [9]. A small number of limited quality randomised controlled trials evaluating digital self-management solutions have observed benefits in health-related quality of life and activity levels [10].

The NHS in England has approved a single digital solution (myCOPD) to support COPD self-management. myCOPD is a web-based app that has been shown to improve inhaler use [11] and, through an online pulmonary rehabilitation programme, improve exercise capacity in severe COPD [11]. A feasibility trial of myCOPD suggested potential to reduce readmissions, improve symptoms and reduce inhaler errors following hospitalisation with a COPD exacerbation [12, 13]. However, little is known about myCOPD's role in mild, moderate or recently diagnosed COPD. The endorsement of COPD case-finding is likely to significantly increase the proportion of patients with milder disease. It is therefore important to understand the role of digital self-management tools in this population. We have undertaken a randomised controlled trial comparing myCOPD with usual care in patients with mild, moderate and recently diagnosed COPD in a primary care setting to explore how patients use the app and whether its use can improve the ability to self-manage their condition, as measured by improvements in symptom control and inhaler technique.

\section{Methods}

\section{Study design}

This was a 12-week, open-label, parallel-group, randomised controlled trial of myCOPD compared with usual care in people with mild-moderate COPD or COPD of any severity diagnosed within the last 12 months. The trial was conducted in three sites in England and involved two site visits and one telephone contact. Participants were randomised 1:1 (myCOPD:usual care). Randomisation was conducted via an online system (my mhealth), was stratified by COPD severity and used permuted blocks. Participant blinding was not possible due to the nature of the intervention. Both groups were invited to participate in a sub-study of physical activity monitoring.

The trial received UK research ethics committee and Health Research Authority approval (REC 18/SS/ 0112). The trial was registered on ClinicalTrial.gov (Reference NCT03620630). All subjects provided written informed consent.

\section{Participants}

Participants were identified by clinical teams and recruited between November 2018 and June 2019. Participants were aged 40-80 years with either mild-moderate COPD (forced expiratory volume in $1 \mathrm{~s}$ $\left(\mathrm{FEV}_{1}\right)>50 \%$ predicted and $\mathrm{FEV}_{1}$ /forced vital capacity ratio $\left.<70 \%\right)$ or COPD of any severity diagnosed within the past 12 months. They were required to be current or ex-smokers, have internet access and be able to use a web platform in English. Individuals were unable to take part if they had a COPD exacerbation within 4 weeks before enrolment, were housebound or had another medical condition considered by the investigator to confound study outcomes.

Affiliations: ${ }^{1}$ Hull York Medical School, Hull, UK. ${ }^{2}$ Imperial College London, London, UK. ${ }^{3}$ Hampshire Hospitals NHS Foundation Trust - Basingstoke Hospital, Basingstoke, UK. ${ }^{4}$ West Hertfordshire Hospital NHS Trust - Hemel Hempstead Hospital, Hemel Hempstead, UK. ${ }^{5}$ mymhealth Limited, Bournemouth, UK. 'University of Southampton Faculty of Medicine, Southampton, UK.

Correspondence: Michael G. Crooks, Academic Respiratory Medicine, Institute of Clinical and Applied Health Research, Hull York Medical School, Castle Hill Hospital, Cottingham, HU16 5JQ, UK. E-mail: Michael.crooksahey.nhs.uk 


\section{Intervention}

myCOPD is an online application to support patients with COPD through education, self-monitoring and self-management functions. Participants randomised to the intervention were registered by the healthcare team and then self-activated the app at their convenience using an e-mail link. Once accessed, a "how to use" video provided information on app content and usage. Thereafter, users were able to access the tile platform and utilise all aspects of the app by clicking on each tile and inputting data. Further details about the app and its functions are available in the supplementary figures S1 to S4.

Participants used the app as they wished and did not receive coaching or encouragement from researchers during the study. Participants in the intervention arm had app access for the duration of the study and were granted life-long access on study completion.

Participants randomised to usual care were asked to continue their usual COPD management for the study duration. After study completion, they were offered life-long app access.

\section{Outcomes}

The co-primary outcomes were the 1) between-group difference in mean change in CAT score and 2) proportion of patients with $\geqslant 1$ critical inhaler error at 90 days. The CAT questionnaire is a validated measure of COPD impact on health status with higher scores indicating greater impact [14]. CAT score was recorded at baseline, month 1 , month 2 and end of study.

Inhaler technique was evaluated by two assessors: one blinded and one unblinded to the intervention. The unblinded assessor observed technique at the baseline visit and the blinded assessor at end of study. Inhaler technique was assessed using placebo devices and the seven steps developed by the UK Inhaler Group (UKIG).

Secondary outcomes included: between-group difference in mean change from baseline at study completion in patient activation measurement (PAM) score, a 13-item scale assessing an individual's knowledge, skill and confidence for self-management [15]; self-efficacy for appropriate medication use scale (SEAMS), a validated medication adherence questionnaire assessing medication self-efficacy in chronic disease management [16]; and EuroQol 5 dimensions 5-level questionnaire (EQ5D 5L), a standardised 5-dimensional instrument measuring health-related quality of life in adults [17].

Activity monitoring was undertaken in a subgroup for a 7-day period at baseline and then for 7 days prior to the end of study visit. Participants had step count measured using a fitbit (fitbit inc., San Francisco, CA, USA).

App usage, completion of educational app content and patient satisfaction were also assessed. Exacerbations, PAMs and SEAMS were collected at all timepoints, with all other secondary outcomes collected at two timepoints, baseline and end of study.

\section{Statistical analysis}

The sample size was calculated on estimating the precision of the point estimate (mean difference in CAT score). Sixty participants (30 per group) were required to estimate $95 \%$ confidence interval with precision of \pm 4.3 assuming a standard deviation of 8.4 [14]. Primary analysis used the intention-to-treat (ITT) principle, defined as participants randomised with at least one post-baseline measurement. Participants with missing baseline data were included in ITT analysis, using mean imputation for continuous or binary baseline measurements [18]. For categorical data (PAM level) participants were assigned to the group closest to the mean.

A linear mixed-effects model with a group $\times$ time interaction estimated the mean between-group difference in CAT score at 90 days with $95 \%$ confidence interval and corresponding p-value after adjustment for COPD severity, baseline CAT score and study centre. Participant-level random effects were used to account for repeated-measures correlation. Missing outcome data were assumed to be missing at random (MAR) conditional on the observed data.

For the co-primary outcome critical inhaler errors, up to three inhalers were examined per participant and two analyses performed. Firstly, logistic regression was performed to estimate the odds ratio of $\geqslant 1$ critical error (in $\geqslant 1$ inhaler) at 90 days, adjusted for baseline critical inhaler error, COPD severity and centre. Secondly, a Poisson regression model estimated the relative rate in mean number of inhaler errors (across all the participants' inhalers) between groups, adjusted for baseline inhaler mean errors, COPD severity and centre. Pre-planned sensitivity analysis using controlled multiple imputation for CAT score explored the MAR assumption [19]. A $\pm 20 \%$ difference in unobserved score was assessed for all participants with missingness and only in one arm (repeated for both groups). Inhaler technique sensitivity used a worst-case/best-case analysis for those with missing outcome for $\geqslant 1$ critical error at 90 days. 
PAM score and SEAMS were modelled using the same linear mixed-effects model as CAT score. ANCOVA to estimate mean group difference was used for EQ5D utility score, visual analogue scale (VAS) and activity level. Smoking status and smoking cessation at 90 days were analysed using logistic regression. An ordered logistic regression was used to analyse PAM level. A Poisson regression was fitted to model the exacerbation rate difference between groups. Participants' actual day of study visit was used as the offset. For participants who withdrew during the study day of visit, it was calculated as previous visit day +15 days. All models included adjustment for COPD severity (except smoking cessation), baseline measure and study centre.

A compliance average cause effect (CACE) analysis was performed on CAT score to estimate the intervention effect in those using the app. Adherence was binary and defined as participants randomised to intervention with at least one post-baseline assessment and who used the intervention to the defined threshold. These were determined by assessing app usage over the study and evaluating total days used, events recorded in the app and corresponding change in outcome.

A full statistical analysis plan was written prior to data extraction and is available upon request.

\section{Results}

Patients

Sixty patients were randomised into the study (29 myCOPD and 31 usual care). The key baseline demographics of study participants are summarised in table 1 and subject flow through the study in figure 1 . Baseline values were imputed for three (5.0\%) participants; one for COPD severity, two for SEAMS, PAM score and PAM level, and one for CAT score and EQ5D utility score and VAS.

Although COPD severity in terms of $\mathrm{FEV}_{1} \%$ pred was well balanced between groups, there was imbalance for other clinical factors including: at least one exacerbation in the last 3 months (myCOPD $11(37.9 \%)$ patients versus usual care 3 (9.7\%) patients); sex (11 (37.9\%) males versus 20 (64.5\%) males); CAT score (mean \pm SD $21.5 \pm 8.0$ versus $19.8 \pm 5.3$ ), PAM score $(59.9 \pm 15.9$ versus $69.0 \pm 13.8$ ) and proportion in PAM levels (6 (20.7\%) in highest level versus $13(41.9 \%))$. At least one post-randomisation outcome measurement was available for $58(96.7 \%)$ for the primary outcome CAT score; outcomes assessed only at 90 days were available for $54(90.0 \%)$ participants.

\section{App usage}

App usage data were available for 26 (89.7\%) participants; all 26 were registered for the app but 5 (17.2\%) participants did not activate it. App use profiles for the 26 participants are shown in figure 2. Of the 21 activated users, 18 subjects (86\%) were still using the app in the last month of the trial. The minimum number of app users in a given week was 13 (45\%). Of the 21 participants who activated the app, 20 participants accessed the app on at least 2 other days.

The median time for participants to activate the app was 1 day (interquartile range (IQR) 1-2 days). The myCOPD app was used on a mean 44 days (SD 31.6 days, median 42 days, IQR 17-75 days) corresponding to an average use over 9 separate weeks by a participant. The total mean number of app activities recorded was 87.8 (SD 118.7, median 67.5, IQR 4-111), with half of these (42.5) recording clinical scores and half (45.3) accessing educational videos. 24 (82.8\%) participants provided feedback on how useful they found the app (see supplementary material).

To perform CACE analysis, adherence definitions were constructed based on observed use profiles in figure 2. Six definitions were constructed, three for total use and three for sustained use, reflecting the spectrum of app use in a linear fashion. Total use definitions were: activating the app and at least one activity; accessing the app on $>30$ days; and accessing the app on at least 60 days. Sustained use definitions were: an activity in the app in at least $50 \%$ of trial weeks; an activity in the app in at least $75 \%$ of trial weeks; and an activity in the app in at least $90 \%$ of the weeks in the first half and $90 \%$ of the weeks in the second half of the trial.

\section{Primary outcomes}

\section{CAT score}

The adjusted mean intervention group difference at 90 days was lower in the myCOPD arm by a mean of -1.27 (95\% CI $-4.47-1.92, \mathrm{p}=0.44, \mathrm{n}=58$ ). The mean CAT score reduced from $21.5 \pm 8.0$ at baseline to $19.2 \pm 9.0$ at 90 days in the myCOPD arm (unadjusted change at 90 days $-1.8 \pm 5.8, n=24$ ). In the usual care arm, the mean CAT score changed from $19.8 \pm 5.4$ at baseline to $19.8 \pm 7.5$ at 90 days (unadjusted change at 90 days $0.03 \pm 5.5, \mathrm{n}=30$ ). The mean change from baseline by treatment arm is shown in figure 3 .

For adherence adjusted analysis for total use (table 2) the adjusted CACE analysis intervention group differences in CAT score at 90 days for myCOPD were: -1.63 (95\% CI $-5.56-2.30, \mathrm{n}$ adhered=18); 


\begin{tabular}{|c|c|c|c|}
\hline \multirow[t]{2}{*}{ Baseline characteristic } & \multicolumn{2}{|c|}{ Treatment } & \multirow[t]{2}{*}{ Total } \\
\hline & myCOPD & Standard care & \\
\hline Subjects n & 29 & 31 & 60 \\
\hline \multicolumn{4}{|l|}{ COPD severity } \\
\hline Mild & $7(24.1)$ & $7(22.6)$ & $14(23.3)$ \\
\hline Moderate & $22(75.9)$ & $24^{\#}(77.4)^{\#}$ & $46^{\#}(76.7)^{\#}$ \\
\hline Age years & $65.9 \pm 7.3$ & $66.4 \pm 7.0$ & $66.1 \pm 7.1$ \\
\hline Years since COPD diagnosis & $7.9 \pm 6.9$ & $6.1 \pm 5.9$ & $7.0 \pm 6.4$ \\
\hline \multicolumn{4}{|l|}{ Gender } \\
\hline Female & $18(62.1)$ & 11 (35.5) & 29 (48.3) \\
\hline Male & 11 (37.9) & $20(64.5)$ & 31 (51.7) \\
\hline Current smoker & $7(24.1)$ & $9(29.0)$ & $16(26.7)$ \\
\hline Years of smoking & $39.0 \pm 11.0$ & $38.6 \pm 12.5$ & $38.8 \pm 11.7$ \\
\hline$\geqslant 1$ exacerbation (past 3 months) & $11(37.9)$ & 3 (9.7) & $14(25.0)$ \\
\hline Treated with antibiotics & $10(34.5)$ & $2(6.5)$ & $12(20.0)$ \\
\hline Treated with steroids & $8(27.6)$ & $3(9.7)$ & $11(18.3)$ \\
\hline Requiring emergency department attendance & $0(0.0)$ & $1(3.2)$ & $1(1.7)$ \\
\hline Requiring hospitalisation & $0(0.0)$ & $1(3.2)$ & $1(1.7)$ \\
\hline Requiring intensive therapy unit admission & $0(0.0)$ & $0(0.0)$ & $0(0.0)$ \\
\hline Total duration of stay days & $0.0 \pm 0.0$ & $0.8 \pm 1.5$ & $0.2 \pm 0.8$ \\
\hline CAT & $21.5 \pm 8.0)$ & $19.8^{\#} \pm 5.3^{\#}$ & $20.6^{\#} \pm 6.7^{\#}$ \\
\hline EQ5D 5L Index Score & $0.6 \pm 0.31$ & $0.7(0.2)$ & $0.6(0.3)$ \\
\hline EQ5D 5L VAS & $61.9 \pm 20.6$ & $63.3^{\#} \pm 19.7^{\#}$ & $62.6^{\#} \pm 20.0^{\#}$ \\
\hline PAM & $59.9 \pm 15.91$ & $\left.69.0^{\#} \pm 13.8\right)^{\#}$ & $64.6^{\#} \pm 15.4^{\#}$ \\
\hline \multicolumn{4}{|l|}{ PAM level } \\
\hline 1 & 6 (20.7) & $1(3.2)$ & $7(11.7)$ \\
\hline 2 & $8(27.6)$ & $2(6.5)$ & $10(16.7)$ \\
\hline 3 & $9(31.0)$ & $15^{\#}(48.4)^{\#}$ & $24^{\#}(40.0)^{\#}$ \\
\hline 4 & 6 (20.7) & $13(41.9)$ & 19 (31.7) \\
\hline SEAMS & $32.8^{\#} \pm 5.7^{\#}$ & $33.8 \pm 4.9$ & $33.3^{\#} \pm 5.3^{\#}$ \\
\hline Individuals with $\geqslant 1$ critical error - inhaler technique & $21(72.4)$ & $18(58.1)$ & $39(65.0)$ \\
\hline Average errors per device & $1.1 \pm 1.3$ & $1.0 \pm 1.1$ & $1.0 \pm 1.2$ \\
\hline Daily step count & $4948.7 \pm 1667.6$ & $9060.1 \pm 5135.1$ & $7591.8 \pm 4611.1$ \\
\hline \multicolumn{4}{|c|}{$\begin{array}{l}\text { Data are presented as } \mathrm{n}(\%) \text { or mean } \pm \mathrm{SD} \text {, unless otherwise stated. The denominator for percentages is the } \\
\text { number randomised into the study. Three participants with missing data have been included in this table; } \\
\# \text { denotes variables that were imputed as a result. CAT: COPD assessment test; EQ5D } 5 \mathrm{~L} \text { : EuroQol } 5 \\
\text { dimensions } 5 \text {-level questionnaire; VAS: visual analogue scale; PAM: patient activation measurement; } \\
\text { SEAMS: self-efficacy for appropriate medication use scale. }\end{array}$} \\
\hline
\end{tabular}

-2.47 (95\% CI $-8.46-3.53, \mathrm{n}=12$ ); and -4.28 (95\% CI $-15.00-6.43, \mathrm{n}=7$ ) for each total use definition respectively.

There was an estimated -0.22 (95\% CI -0.74-0.31) decrease in score for every 7-day increase in app use, adjusted for baseline CAT score, COPD severity and site. The adjusted CACE estimates for sustained use definitions were: $-2.13(95 \%$ CI $-7.24-2.98, \mathrm{n}=14$ ) for $50 \%$ of weeks active; -2.47 (95\% CI $-8.46-3.53$, $\mathrm{n}=12$ ) for $75 \%$ of weeks active; and $-2.93(95 \%$ CI $-9.97-4.10, \mathrm{n}=10)$ for $90 \%$ of weeks in first and second halves active.

\section{Inhaler technique}

For the ITT analysis the odds of $\geqslant 1$ critical error in the myCOPD arm was lower compared to usual care with an adjusted odds ratio of 0.30 (95\% CI $0.09-1.06, \mathrm{p}=0.061, \mathrm{n}=54$ ). The adjusted mean count of inhaler errors in myCOPD were 0.97 (95\% CI $0.52-1.81, \mathrm{p}=0.93$ ) times the inhaler errors of those in the usual care arm.

\section{Secondary outcomes}

The adjusted mean difference in PAM score at 90 days was -0.98 (95\% CI -8.22-6.26). There was a higher relative increase in the proportion of participants moving to the highest PAM level (from baseline 


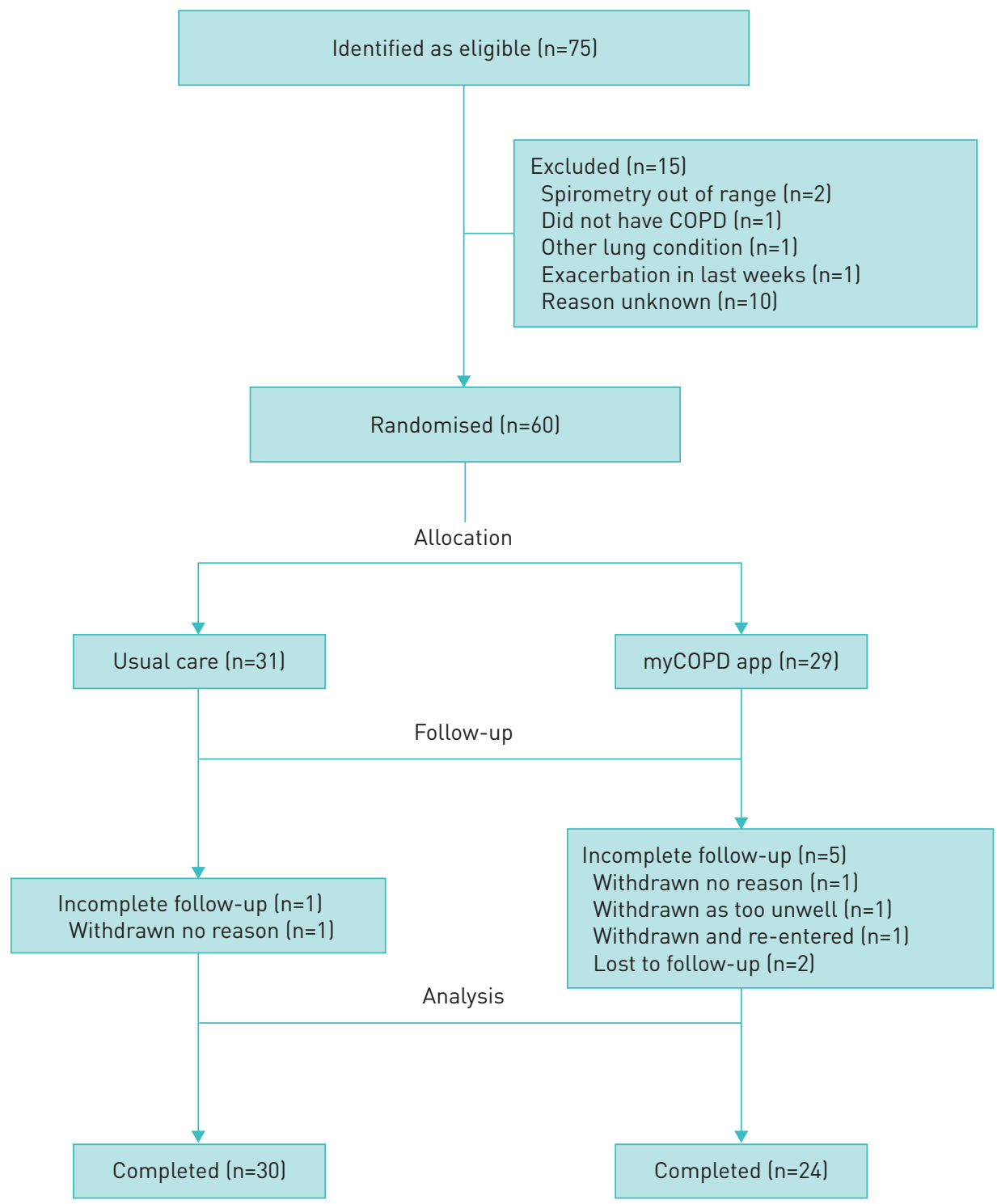

FIGURE 1 CONSORT flow diagram for the study. The participant re-entered in myCOPD was still excluded from analysis as 6 months had elapsed between baseline and post-baseline assessments.

to day 90) in the myCOPD group compared to usual care, 1.4 and 0.93 respectively (table 3 ). The adjusted odds ratio for being in a higher PAM level at 90 days was $1.65(0.46 ; 5.85)$ in favour of myCOPD.

Mean SEAMS scores were similar between groups at all timepoints, the adjusted mean intervention difference at 90 days was $1.48(-1.47 ; 4.42)$. The 90 -day adjusted mean EQ5D-5L utility score intervention difference was $-0.04(-0.12 ; 0.05)$ and the 90 -day adjusted mean VAS score intervention difference was $0.86(-9.46 ; 11.18)$.

Fifteen exacerbations were recorded in the 3 months prior to study baseline (12 myCOPD and 3 usual care). Twenty-nine exacerbations were recorded during the study (18 myCOPD group and 11 usual care). Three $(10.3 \%)$ exacerbation events required emergency department attendance (2 myCOPD and 1 usual care) and $3(10.3 \%)$ required hospitalisation (1 myCOPD and 2 usual care). The number of exacerbations at baseline and throughout the study and their treatment are displayed in table 4 .

Fourteen (23.3\%) participants volunteered for the activity sub-study (5 (35.7\%) myCOPD and 9 (64.3\%) usual care). The mean number of steps per day at baseline was 4948.7 (SD 1667.6, n=5) for myCOPD and 9060 (5135.1, n=9) for usual care. At the end of the study the mean number of steps per day was 5458.3 $(2266.4, \mathrm{n}=4)$ for myCOPD and $10762(7199.2, \mathrm{n}=9)$ for usual care. The adjusted mean daily step count in the myCOPD arm was -2252 steps lower ( -10433.8 to 5927.9$)$. 


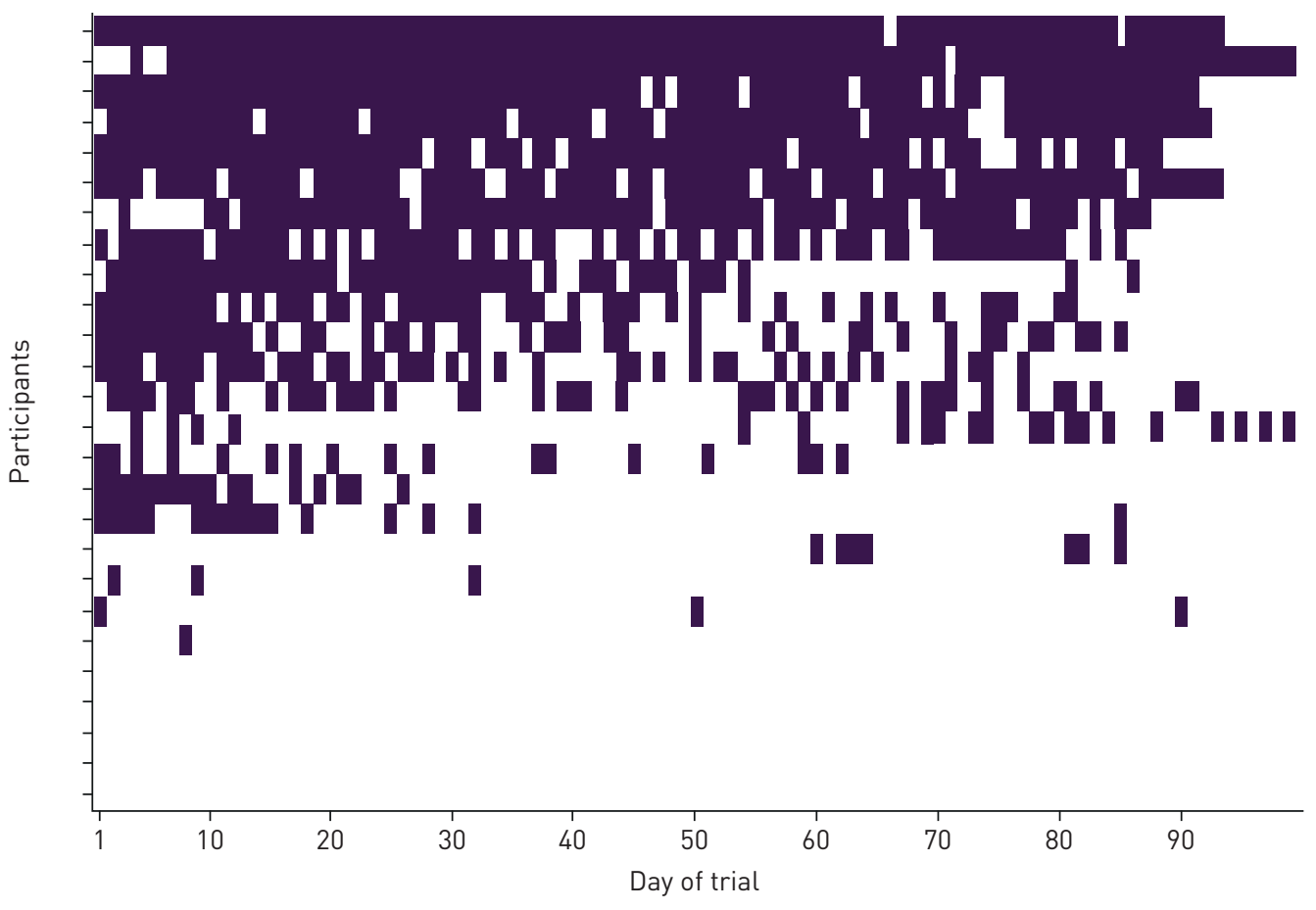

FIGURE 2 Participants' profiles of using the app at least once per day over the trial period. Data shown here were available for 26 of the 29 participants; first day is defined as baseline visit. Each row in the figure corresponds to the profile of a participant where a coloured square means an activity, e.g. watched a video or reported symptom score, recorded in the app for that day.

\section{Safety outcomes}

Over the study period, 15 adverse events were reported by 12 (20.0\%) participants (5 from myCOPD and 7 from usual care). Two participants, both in usual care, reported multiple adverse events. No serious adverse events were reported during the study.

\section{Discussion}

This is the first randomised controlled trial of the myCOPD app in mild, moderate and recently diagnosed COPD. The study demonstrated good engagement with the app self-management intervention with patterns of sustained use at 90 days seen. Signals of improvement in inhaler technique and disease activity score were found with evidence for increased impact in patients who used the app more often. Our findings provide important insights into the role and optimum use profile for myCOPD with a clear association between increased app use and clinically meaningful benefits in CAT score.

Understanding patterns of engagement and adherence is important when considering an intervention's effectiveness and place in clinical care. Given the incomplete understanding of the optimal frequency or

FIGURE 3 Mean change in COPD assessment test (CAT) score for each timepoint compared to baseline. Participants are included at each timepoint if a CAT score was recorded. For myCOPD there are 29 participants included at baseline, 25 at month 1 and 24 at month 2 and end of study (EOS). For usual care there are 31 participants included at baseline, 30 at month 1, 29 at month 2 and 30 at end of study.

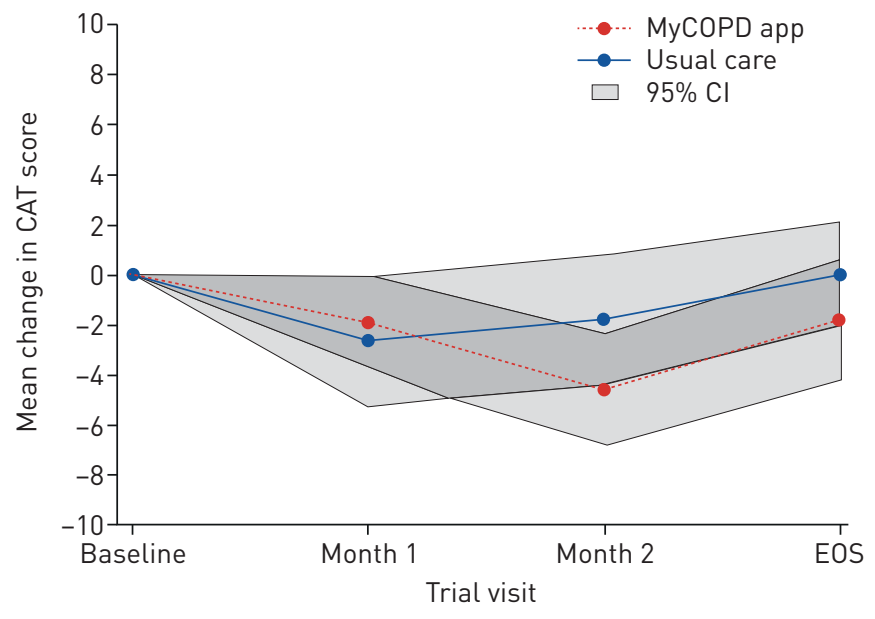


TABLE 2 Treatment difference in COPD assessment test (CAT) score at 90 days for different

definitions of adherence

\begin{tabular}{|c|c|c|c|c|}
\hline \multicolumn{2}{|l|}{ Usage definitions } & \multirow{3}{*}{$\begin{array}{c}\text { Active users } \\
18 \\
12\end{array}$} & \multirow{2}{*}{$\begin{array}{c}\begin{array}{c}\text { Adjusted treatment } \\
\text { estimate }\end{array} \\
-1.63\end{array}$} & \multirow{2}{*}{$\begin{array}{c}\mathbf{9 5 \%} \text { confidence interval } \\
-5.56-2.30\end{array}$} \\
\hline Total usage & Activated app & & & \\
\hline & $>30$ days & & -2.47 & $-8.46-3.53$ \\
\hline & $\geqslant 60$ days & 7 & -4.28 & $-15.00-6.43$ \\
\hline \multirow[t]{3}{*}{ Sustained usage } & $50 \%$ weeks active & 14 & -2.13 & $-7.24-2.98$ \\
\hline & $75 \%$ weeks active & 12 & -2.47 & $-8.46-3.53$ \\
\hline & $90 \%$ first, $90 \%$ second & 10 & -2.93 & $-9.97-4.10$ \\
\hline
\end{tabular}

timing of app use, it was not possible to benchmark optimal adherence with myCOPD in this population a priori. The first stage in digitally supported self-management is user engagement. Participants in this trial received no coaching or prompts to engage with myCOPD. This allowed us to understand how patients interacted with the app and explore the relationship between use and clinical outcomes. An integrative definition of engagement includes assessment of the extent of use and the user's subjective experience [20]. We characterised the extent of app use based on how many times participants interacted with the app during the study (total use definition) and the patterns of use during the study (sustained use definition). A previous survey of health app users reported that almost three-quarters of people that download an app will have stopped using it before their 10th use [21]. myCOPD use was high with 65\% of participants allocated to the app having used it $>10$ times. Importantly, baseline PAM level did not correlate with app engagement (data not shown), and therefore those with low baseline activation levels are as likely to engage with myCOPD as those already activated.

Although we observed high levels of app use throughout the study, there was a trend towards reduced use frequency over the trial duration. This phenomenon, termed the law of attrition, is well described in trials of digital health solutions and leads to underestimation of an interventions efficacy when undertaking ITT

TABLE 3 Results of all regression analysis and 90-day estimates for primary and secondary outcomes

\begin{tabular}{|c|c|c|c|c|c|c|}
\hline \multirow[t]{2}{*}{ Effectiveness outcomes } & \multicolumn{2}{|c|}{ 90-day change from baseline } & \multirow{2}{*}{$\begin{array}{l}\text { Regression } \\
\text { sample size }\end{array}$} & \multirow{2}{*}{$\begin{array}{l}\text { Adjusted group } \\
\text { difference }\end{array}$} & \multirow{2}{*}{$\begin{array}{l}95 \% \text { confidence } \\
\text { interval }\end{array}$} & \multirow[t]{2}{*}{$p$-value } \\
\hline & myCOPD $\#$ & Usual care & & & & \\
\hline \multicolumn{7}{|l|}{ Primary outcomes } \\
\hline$\geqslant 1$ inhaler error & $-0.3(0.70)$ & $0.1(0.71)$ & 54 & OR: 0.30 & $0.09-1.06$ & 0.061 \\
\hline Average inhaler errors & $-0.3(1.61)$ & $-0.1(1.20)$ & 54 & IRR: 0.97 & $0.52-1.81$ & 0.928 \\
\hline \multicolumn{7}{|l|}{ Secondary outcomes } \\
\hline SEAMS & $1.0(0.00)$ & $0.0(-3.00)$ & 58 & MD: 0.33 & $-2.22-2.87$ & \\
\hline EQ5D score & $0.1(0.23)$ & $0.0(0.18)$ & 54 & MD: -0.04 & $-0.12-0.05$ & \\
\hline EQ5D VAS & $62.0(21.35)$ & $60.9(19.92)$ & 53 & MD: 0.86 & $-9.46-11.18$ & \\
\hline Exacerbations & $0.2(1.28)$ & $0.2(0.72)$ & 60 & IRR: 2.55 & $1.17-5.54$ & \\
\hline $\begin{array}{l}\text { Activity level (mean daily } \\
\text { steps) }\end{array}$ & $\begin{array}{c}226.8 \\
(5680.38)\end{array}$ & $\begin{array}{c}11915.9(37 \\
447.87)\end{array}$ & 13 & MD: -2252.94 & $-10433.77-5927.88$ & \\
\hline
\end{tabular}

All regression models included adjustment for baseline values, COPD severity and centre. $\mathrm{n}$ in 90 -day observed outcome is defined as participants who were at the final study visit. CAT: COPD assessment test; MD: mean difference; OR: odds ratio; IRR: incidence rate ratio; PAM: patient activation measurement; SEAMS: self-efficacy for appropriate medication use scale; EQ5D: EuroQol 5 dimensions; EQ5D VAS: EuroQol 5 dimensions visual analogue scale. ${ }^{\#}: \mathrm{n}=24 ;{ }^{~ "}: \mathrm{n}=30$. Example interpretation: $M D-A$ difference of -1.27 means after adjustment for baseline values, COPD severity and centre the mean CAT score was 1.27 lower in myCOPD. IRR - A difference of 0.97 means after adjustment for baseline values, COPD severity and centre the rate of average inhaler errors for myCOPD was 0.97 times the rate of average inhaler errors for usual care. OR - A difference of 0.30 means after adjustment, the odds of $\geqslant 1$ inhaler error in myCOPD were 0.30 times the odds of $\geqslant 1$ inhaler error in usual care. 


\section{TABLE 4 Numbers of exacerbations, and treatment, at baseline and throughout the study}

\begin{tabular}{|c|c|c|c|c|c|c|c|c|c|c|}
\hline \multirow{3}{*}{$\begin{array}{l}\text { Timepoint } \\
\text { Treatment }\end{array}$} & \multicolumn{2}{|c|}{ Exacerbations } & \multicolumn{8}{|c|}{ Treatment } \\
\hline & \multirow[b]{2}{*}{$\mathbf{N}$} & \multirow[b]{2}{*}{$n$ events } & \multicolumn{2}{|c|}{ Antibiotics } & \multicolumn{2}{|c|}{ Steroids } & \multicolumn{2}{|r|}{ Both } & \multicolumn{2}{|r|}{ None } \\
\hline & & & $\mathbf{N}$ & n events & $\mathbf{N}$ & n events & $\mathbf{N}$ & n events & $\mathbf{N}$ & n events \\
\hline \multicolumn{11}{|l|}{ Baseline } \\
\hline myCOPD & 11 & 12 & 3 & 3 & 1 & 2 & 7 & 7 & 0 & 0 \\
\hline Usual care & 3 & 3 & 0 & 0 & 2 & 2 & 1 & 1 & 0 & 0 \\
\hline \multicolumn{11}{|l|}{ Post-baseline } \\
\hline myCOPD & 13 & 18 & 6 & 8 & 2 & 2 & 4 & 6 & 2 & 2 \\
\hline Usual care & 8 & 11 & 2 & 2 & 1 & 1 & 6 & 6 & 1 & 2 \\
\hline
\end{tabular}

analysis [22]. An alternative explanation in our trial is that myCOPD users accessing the pulmonary rehabilitation programme in the early stages of the trial will have completed it in the second month, contributing to a reduction in app usage in the later trial stages.

Both total and sustained myCOPD use definitions were associated with greater improvements in CAT score during the trial. The reduction in CAT score in those using the app on $>30$ days (total use) or for at least $50 \%$ of trial weeks (sustained use) exceeded the minimum clinically important difference and was similar in magnitude to the reduction observed in clinical trials of inhaled bronchodilators [23]. This improvement is likely multifactorial with improved self-management, participation in the pulmonary rehabilitation programme and improved inhaler technique possible explanations [11].

The clear pattern of greater benefit with increased myCOPD use is consistent with previous studies of digital behaviour change interventions including smoking cessation and weight loss [24, 25]. This is important when considering implementation of a digital solution like myCOPD in clinical practice. Patients should be counselled about the importance of regular and sustained app use, and services should be designed to support engagement.

While this study aimed to evaluate the effectiveness of myCOPD to support participant's self-management, the small sample size and marked phenotypic difference between groups limited our ability to demonstrate statistically significant differences in our primary outcomes at 90 days. The marked between-group differences at baseline favoured within group assessment of treatment effect rather than direct comparison. Despite stratified randomisation, the groups in this study represent two distinct COPD populations with different clinical phenotypes. The myCOPD group were predominantly female with a high baseline symptom burden, significantly lower physical activity level and had a higher proportion of patients in the lowest activation levels. The myCOPD group had a significantly higher exacerbation frequency at baseline when compared to the usual care group, a trait which continued throughout the trial. Despite the higher number of exacerbations in the myCOPD group, the number of exacerbations resulting in emergency department attendance or hospital admission was no different between groups.

Physical activity monitoring was undertaken as a voluntary sub-study. More participants in the usual care group volunteered and were markedly more active at baseline than those that chose to take part in the myCOPD arm. Both groups demonstrated an improvement in physical activity during the trial. Those undertaking activity monitoring will have received biofeedback from the activity monitor, potentially promoting activity and impacting clinical outcomes for these participants.

Digital solutions to support self-management and improve patient outcomes have never been more important. This trial was undertaken before the emergence of a novel coronavirus that has swept the globe and fundamentally changed the way that healthcare is delivered. Digital technologies now play a central role in delivering healthcare. COPD patients are considered vulnerable to coronavirus disease 2019 (COVID-19) and therefore minimising health service contacts and delivering care remotely is attractive. MyCOPD has the potential to support patient care through encouraging patients to take a more active role in self-management, improving inhaler techniques and improving exercise capacity that will have been lost through shielding [11]. A larger, adequately powered trial is needed to confirm the effectiveness of myCOPD in this setting.

\section{Conclusion}

This trial provides important insights into how patients with mild-moderate COPD use the app myCOPD. Signals of potential efficacy were seen in the study which became more apparent with sustained use 
patterns, but the ability to demonstrate statistically significant differences between groups was impaired by the small sample size, law of attrition and the marked phenotypic difference at baseline typical of a preliminary small study. The potential to generate clinically important improvements in CAT score and inhaler technique with a cheap, scalable technology suggest larger scale use and evidence generation are required to understand significant benefits for patients and services over the long term.

Conflict of interest: M.G. Crooks has nothing to disclose. J. Elkes has nothing to disclose. W. Storrar has nothing to disclose. K. Roy has nothing to disclose. M. North is an employee of mymhealth Limited. He reports grants from SBRI during the conduct of the study and personal fees from mymhealth Limited outside the submitted work. A. Blythin reports grants from Innovate UK during the conduct of the study and is an employee of mymhealth Limited. A. Watson has nothing to disclose. V. Cornelius has nothing to disclose. T. Wilkinson is the founder and directior of MyMHealth. $\mathrm{He}$ reports grants from Innovate UK during the conduct of the study; and personal fees and other support from MyMHealth, grants from GSK, grants and personal fees from AstraZeneca and Synairgen, and personal fees from BI, outside the submitted work.

Support statement: The study was funded by a UKRI Innovate UK Grant to mymhealth. Funding information for this article has been deposited with the Crossref Funder Registry.

\section{References}

1 Alwan A. Global Status Report on Non-Communicable Diseases. WHO, 2014. https://apps.who.int/iris/bitstream/ handle/10665/148114/9789241564854_eng.pdf?sequence=1 Date last accessed: 26 June 2020.

2 Snell N, Strachan D, Hubbard R, et al. S32 Epidemiology of chronic obstructive pulmonary disease (COPD) in the UK: findings from the British Lung Foundation's 'respiratory health of the nation' project. Thorax 2016; 71: A20.

3 NICE guideline [NG115] Chronic obstructive pulmonary disease in over 16s: diagnosis and management. https:// www.nice.org.uk/guidance/ng115/resources/chronic-obstructive-pulmonary-disease-in-over-16s-diagnosis-andmanagement-pdf-66141600098245 Published 5 December 2018. Date last updated: 26 July 2019.

4 Department of Health. An outcomes strategy for COPD and asthma: NHS companion document. Impact report, 2012. https://assets.publishing.service.gov.uk/government/uploads/system/uploads/attachment_data/file/216531/dh_ 134001.pdf

5 Wilkinson TMA, Donaldson GC, Hurst JR, et al. Early therapy improves outcomes of exacerbations of chronic obstructive pulmonary disease. Am J Respir Crit Care Med 2004; 169: 1298-1303.

6 Williams NP, Coombs NA, Johnson MJ, et al. Seasonality, risk factors and burden of community-acquired pneumonia in COPD patients: a population database study using linked health care records. Int J Chron Obstruct Pulmon Dis 2017; 12: 313-322.

7 Zwerink M, Brusse-Keizer M, van der Valk PD, et al. Self management for patients with chronic obstructive pulmonary disease. Cochrane Database Syst Rev 2014; 2014: CD002990.

8 Griffiths F, Lindenmeyer A, Powell J, et al. Why are health care interventions delivered over the internet? A systematic review of the published literature. J Med Internet Res 2006; 8: e10.

9 Slevin P, Kessie T, Cullen J, et al. A qualitative study of chronic obstructive pulmonary disease patient perceptions of the barriers and facilitators to adopting digital health technology. Digit Health 2019; 5: 2055207619871729.

10 McCabe C, McCann M, Brady AM. Computer and mobile technology interventions for self-management in chronic obstructive pulmonary disease. Cochrane Database Syst Rev 2017; 5: CD011425.

11 Bourne S, DeVos R, North M, et al. Online versus face-to-face pulmonary rehabilitation for patients with chronic obstructive pulmonary disease: randomised controlled trial. BMJ Open 2017; 7: e014580.

12 North M, Bourne S, Green B, et al. P238 A randomised controlled feasibility trial of an E-health platform supported care vs usual care after exacerbation of COPD.(RESCUE COPD). Thorax 2018; 73: A231.

13 North M, Bourne S, Green B, et al. A randomised controlled feasibility trial of E-health application supported care vs usual care after exacerbation of COPD: the RESCUE trial. NPJ Digit Med 2020; in press.

14 Jones PW, Harding G, Berry P, et al. Development and first validation of the COPD Assessment Test. Eur Respir J 2009; 34: 648-654.

15 Hibbard JH, Stockard J, Mahoney ER, et al. Development of the Patient Activation Measure (PAM): conceptualizing and measuring activation in patients and consumers. Health Serv Res 2004; 39: 4 Pt. 1, 1005-1026.

16 Risser J, Jacobson TA, Kripalani S. Development and psychometric evaluation of the Self-efficacy for Appropriate Medication Use Scale (SEAMS) in low-literacy patients with chronic disease. J Nurs Meas 2007; 15: 203-219.

17 Nolan CM, Longworth L, Lord J, et al. The EQ-5D-5L health status questionnaire in COPD: validity, responsiveness and minimum important difference. Thorax 2016; 71: 493-500.

18 White IR, Thompson SG. Adjusting for partially missing baseline measurements in randomized trials. Stat Med 2005; 24: 993-1007.

19 Cro S, Morris TP, Kenward MG, et al. Sensitivity analysis for clinical trials with missing continuous outcome data using controlled multiple imputation: a practical guide. Stat Med 2020; 39: 2815-2842.

20 Perski O, Blandford A, West R, et al. Conceptualising engagement with digital behaviour change interventions: a systematic review using principles from critical interpretive synthesis. Transl Behav Med 2017; 7: 254-267.

21 Consumer Health Information Corporation. Motivating patients to use smartphone health apps. www.prweb.com/ releases/2011/04/prweb5268884.htm Date last accessed: 26 June 2020. Date last updated: 26 June 2020.

22 Eysenbach G. The Law of Attrition. J Med Internet Res 2005; 7: e11.

23 Kon SS, Canavan JL, Jones SE, et al. Minimum clinically important difference for the COPD Assessment Test: a prospective analysis. Lancet Respir Med 2014; 2: 195-203.

24 Cobb NK, Graham AL, Bock BC, et al. Initial evaluation of a real-world Internet smoking cessation system. Nicotine Tob Res 2005; 7: 207-216.

25 Tate DF, Wing RR, Winett RA. Using Internet technology to deliver a behavioral weight loss program. JAMA 2001; 285: 1172-1177. 